\title{
Incidencia de la crisis en el empleo extranjero. Evidencias a partir de las transiciones laborales de trabajadores indefinidos
}

\author{
Nerea Zugasti Mutilva \\ Universidad Pública de Navarra. Departamento de Trabajo Social \\ nerea.zugasti@unavarra.es
}

\section{Resumen}

Este artículo tiene como objetivo la realización de un análisis de las transiciones laborales de la población extranjera que ha accedido a un contrato indefinido. El estudio se centrará en el periodo 2006-2010, tomando en consideración los últimos años del ciclo de bonanza económica más reciente en España y los primeros de la crisis. Se emplearán las versiones transversal y longitudinal de la Encuesta de Población Activa, facilitada por el Instituto Nacional de Estadística.

Los resultados dan cuenta de las dificultades de acceso a los empleos estables que las personas extranjeras experimentan en el mercado de trabajo. Asimismo, muestran una mayor propensión que la población española a realizar trayectorias descendentes. Ser mujer, joven, emplearse en la construcción, tener un empleo no cualificado y un contrato de poca duración son factores de riesgo a la hora de entender el paso de personas no nacionales hacia la temporalidad o el desempleo desde un contrato indefinido. Sin embargo, encontramos también resistencias en el empleo estable entre la población extranjera, así como pautas de convergencia con la población española en indicadores positivos. En un momento de conmoción en el mercado de trabajo, aumenta el número de personas extranjeras que continúan empleadas con un contrato indefinido.

Palabras clave: estabilidad contractual; inmigrantes; mercado de trabajo; movilidad laboral; recesión; trabajadores extranjeros; crisis económica

\section{Abstract. Impact of the crisis on the employment of foreigners: Evidence based on labor transitions of permanent workers}

This paper analyzes labor market transitions among foreigners with permanent contracts over the period 2006-2010, taking into account the last years of the economic boom in Spain and the initial years of the crisis. The study uses data from the cross-sectional and longitudinal versions of the Labor Force Survey conducted by the National Statistics Institute of Spain. The results show that foreigners face challenges in finding stable jobs in the 
labor market and have a greater propensity for downward trajectories than the Spanish. Being female, young, employed in the construction sector, having an unskilled job and a shortterm contract are risk factors that explain the passage of non-nationals from permanent to temporary employment or unemployment. However, we find that foreigners remain in stable employment as well as patterns that converge with the Spanish population in positive indicators. In the midst of a labor market crisis, there is an increasing number of foreigners who remain in permanent employment.

Keywords: contractual stability; immigrants; job market; labor mobility; recession; foreign workers; economic crisis

\author{
Sumario \\ 1. Introducción 6. ¿Cuál es el perfil de la persona \\ 2. Marco teórico del estudio \\ 3. Metodología \\ 4. El punto de partida. Una reflexión \\ de empleo \\ 5. La movilidad desde la situación \\ extranjera contratada bajo la modalidad \\ de contrato indefinido? \\ 7. ¿Quiénes tienen más probabilidad \\ de perder su contrato indefinido? \\ 8. Conclusiones \\ Referencias bibliográficas \\ de contratación indefinida \\ Anexo estadístico
} sobre las implicaciones de la destrucción

\title{
1. Introducción
}

Este artículo tiene como objetivo estudiar las transiciones ${ }^{1}$ laborales de las personas extranjeras que se encuentran empleadas bajo la modalidad de contrato indefinido en España durante un periodo de crisis. Es decir, se abordará el análisis de un grupo específico dentro de la población extranjera. Nos centraremos en el subcolectivo que ha conseguido una cierta estabilidad en el mercado de trabajo medida a través del tipo de contrato. Planteamos un análisis centrado en los años 2006-2010, analizando así la primera fase de una larga crisis, primero, económica; después, del empleo, y, en último término, social (Laparra y Pérez, 2012).

La cuestión es relevante por dos razones. En primer lugar, porque la perspectiva más frecuentemente utilizada para el abordaje de la situación de la población extranjera en el mercado de trabajo en España se refiere a fotos fijas en el tiempo. En contraposición, en esta propuesta, se recurre a una combinación de análisis de tipo transversal con otras propuestas de tipo longitudinal, que permite dar luz a las variaciones y observar la dirección de los cambios. En segundo lugar, porque la situación de aquellas personas extranjeras que han conseguido acceder a una posición de ventaja en el mercado de trabajo, en

1. El concepto de transición puede ser definido como cambios empíricos en la situación de los trabajadores analizados ex post y que nos orientan sobre las probabilidades de que ese tipo de itinerarios sean recorridos por los distintos colectivos analizados (Frade y Darmon, 2004; Laparra, 2006). 
este caso medida por la consecución de un contrato indefinido, ha sido poco analizada en el contexto español. Los análisis sobre inmigración se han centrado en la muy relevante labor de sacar a la luz las situaciones de precariedad laboral de la población extranjera. Sin embargo, entendemos también que el avance del fenómeno migratorio en España hace necesario abordar las situaciones de subcolectivos asentados en el mercado de trabajo. En este caso, personas que han conseguido escapar a la trampa de la precariedad medida a través de la tipología de contrato.

Por un lado, la propuesta parte de la premisa de que el empleo de calidad es un instrumento valioso que facilita la incorporación social de las personas. Por otro lado, se entiende que el estudio de las transiciones constituye un importante reflejo de los procesos de integración en el ámbito laboral de la población inmigrante. La movilidad laboral descendente de la población extranjera supondrá, sin duda, una pérdida de los logros de integración conseguidos. El bloqueo o el mantenimiento en situaciones poco favorables podrán ser leídos como un cierre social. Sin embargo, la movilidad ascendente o el mantenimiento en posiciones ventajosas en el actual contexto supondrán un avance claro en términos de incorporación social.

En esta propuesta, se realiza una revisión de los principales supuestos teóricos para entender las desigualdades en el acceso y el mantenimiento del empleo estable entre nacionales y no nacionales. Seguidamente, se desarrolla la metodología del estudio. Se continúa planteando, como contexto del análisis, una reflexión sobre la destrucción de empleos temporales y estables en el periodo de crisis económica. Posteriormente, se estudian las pautas de movilidad de trabajadores con contrato indefinido de nacionalidad extranjera y española, para desarrollar seguidamente el perfil del empleo estable extranjero en España. Se pormenorizan también los determinantes en la pérdida del empleo indefinido y, finalmente, se desarrollan las conclusiones del análisis.

\section{Marco teórico del estudio}

La cuestión de la integración laboral y de la movilidad de la población inmigrante $^{2}$ ha sido analizada desde diversas perspectivas teóricas. El enfoque neoclásico en el estudio de los movimientos poblacionales entendía los movimientos migratorios como el resultado de una decisión individual y racional de los actores. Los individuos son entendidos como seres libres que optan por la alternativa que les permita maximizar los beneficios con el menor coste posible. Es decir, la decisión de permanecer en el lugar de origen o de desplazarse es la consecuencia de un cálculo basándose en el análisis coste-beneficio (Arango, 2003). La teoría neoclásica mantiene que existe una relación entre la inversión en capital humano y la integración en el mercado de trabajo de la población inmigrante. Estas personas se dirigen hacia lugares donde pueden obtener unas

2. La aproximación a la situación del colectivo inmigrante se realizará fundamentalmente a través de la variable sobre la nacionalidad en la parte empírica del artículo. 
mejores ganancias, pero para ello se ven obligadas a realizar inversiones vinculadas a los costes del desplazamiento, a los costes del aprendizaje del nuevo idioma, de la cultura e incluso a la adquisición de nuevas habilidades profesionales (Massey et al., 1998).

En las teorías del capital humano lo obtenido debe ser explicado a través de los cambios en la productividad de una persona, en sus habilidades y por la acumulación de experiencia (Sørensen, 1977). Según esta perspectiva, las sociedades industriales son meritocráticas y personas con capacidades y habilidades equivalentes tienen las mismas oportunidades y perspectivas de empleo. Dentro de esta tradición, la estructura de los mercados laborales no es vista como fundamental para la explicación de la distribución de recompensas a los individuos a lo largo de su vida laboral, ni para la explicación de su posición laboral (Kalleberg y Sørensen, 1979). Las tesis de la asimilación pronostican una convergencia entre extranjeros y autóctonos conforme aumentan los años de residencia en el país de destino, gracias a la inversión en capital humano por parte de la población extranjera. Las diferencias entre las personas inmigrantes que tienen niveles similares de habilidad y/o formación se deberían a las diferencias en la calidad de la educación recibida en el país de origen, así como a su acomodación a las necesidades del país de acogida (transferibilidad) ${ }^{3}$.

De esta perspectiva, nos interesa su señalamiento de la relación entre la inversión en capital humano (perfil de preparación, años estudiados, experiencia laboral adquirida) y la integración en el mercado de trabajo (salarios, tasas de ocupación y desempleo, acceso a ocupaciones cualificadas y contratos indefinidos) de la población inmigrante. Esta perspectiva se centra en cualidades y competencias de los trabajadores para explicar la posición laboral en la sociedad de acogida. Asimismo, sostiene que los individuos en el mercado de trabajo son recompensados por el valor de la productividad adicional que traen las inversiones en capital humano. Según esta perspectiva, cabría esperar, entonces, una convergencia entre autóctonos y extranjeros en términos de empleo (acceso y mantenimiento en contratos indefinidos).

En contraste con las opiniones de los teóricos neoclásicos, que postulan la existencia de un mercado unificado de mano de obra, el enfoque de la segmentación considera que el mercado de trabajo se divide típicamente en un núcleo y una periferia. Los procesos de asignación ocupacional, formación, ascenso de los salarios y las relaciones entre las distintas posiciones en la escala

3. Esta cuestión se relaciona con la idea del potencial integrador de un determinado territorio. Este concepto nos remite a los factores estructurales que condicionan la integración de las poblaciones extranjeras y autóctonas. Dicho potencial se establece, en primer lugar, a partir de la estructura económica y demográfica del territorio. En segundo lugar, debe ser tenida en cuenta la función que desarrolla en el marco de los flujos migratorios internacionales, que se relaciona con su posición dentro de la división internacional del trabajo. En tercer lugar, se relaciona con la capacidad de sus instituciones para garantizar una acogida adecuada a las personas inmigrantes. Las características del mercado de trabajo y el modelo del estado de bienestar son, por tanto, elementos determinantes del potencial integrador de un territorio (Laparra, 2008). 
de responsabilidades son marcadamente diferentes en ambos sectores. De esta forma, se establece un segmento privilegiado y un segmento precarizado, de baja productividad e intensivo en mano de obra.

Para reflejar la existencia de los diferentes segmentos del mercado, los estudiosos se han referido a mercado «abierto / flexible» frente a mercado "cerrado / inflexible» (Sørensen y Tuma, 1981), a mercados «interno» y "externo» (Althauser, 1989), a los segmentos "primario" $\mathrm{y}$ «secundario» (Doeringer y Piore, 1971). Sin embargo, la lógica básica detrás de todos estos modelos es muy similar. El concepto de los mercados de trabajo duales se ha empleado para poner de relieve las diferencias intergrupales en diferentes submercados o segmentos del mercado de trabajo (Torre, 2011). La población extranjera tendría importantes limitaciones para acceder al estrato laboral privilegiado y quedarían reservados para este colectivo los puestos más precarios y rechazados por la población autóctona (Piore, 1983). Como veremos, existe un importante colectivo que ha conseguido acceder a contratos de tipo indefinido, con lo cual ha saltado una de las trampas de la precariedad. Sin embargo, según esta perspectiva, cabe esperar una mayor propensión a la pérdida de empleo de estas personas «estables» y que vendría explicada por su inserción en puestos no cualificados, más sensibles a las fluctuaciones de ciclo económico. Diversos estudios caracterizan al mercado de trabajo español como segmentado (Huguet, 1999; Toharia, 1997). El énfasis se pone, entonces, en las variables que implican diferencias sociales, como la etnia, el género o la clase (Parella, 2003), o la nacionalidad (Cachón, 2003), con lo cual se remarca la importancia de la estratificación.

Estudios como el de Fernández y Ortega (2008) señalan, para el periodo 1996-2006, que, en comparación con la población nativa, las personas inmigrantes tienen una mayor tasa de desempleo, una mayor sobrecualificación para los puestos que desempeñan, así como mayores tasas de temporalidad. Sin embargo, cinco años después de la inmigración, las tasas de desempleo decrecen a niveles incluso menores que las de los nativos. Por el contrario, la incidencia de la sobrecualificación y de la temporalidad se mantiene constante y no se reduce. Así, señalan que España se ha mostrado eficaz para absorber los flujos migratorios a expensas de condenar a la población inmigrante al trabajo temporal y a puestos no acordes a su cualificación. Este análisis debe enmarcarse en el contexto de un mercado caracterizado por los importantes niveles de temporalidad (Cachón, 1995; Pérez Infante, 2008) y por la baja cualificación de los puestos ofertados (La Caixa, 2009), que se conjugan con unas tasas de paro altas pero muy fluctuantes en función del ciclo económico (González y Pérez, 2006). Desde esta perspectiva, podemos señalar que las transiciones laborales de los sujetos ocurren en el seno de un mercado laboral dual que no sólo determina las condiciones de trabajo en un momento dado, sino también las posibilidades de movilidad social y ocupacional (Centre d'Estudis Sociològics sobre la Vida Quotidiana i el Treball, 2011).

Asimismo, Cachón $(1995,1997)$ plantea un esquema en el que se unen los planos individual y estructural. Señala que los factores institucionales, los económicos y los tecnológicos definen el campo de posibilidades en el que se 
desarrollan las estrategias de empresarios y trabajadores, lo cual determina las estructuras de los diversos mercados que configuran el conjunto del mercado de trabajo. Este autor, en referencia al español, señala que la posición de las personas en esta estructura depende de las «oportunidades de empleo de que disponen» y del «nivel de aceptación por su parte de las condiciones de trabajo", es decir, de su poder de negociación. Las oportunidades de empleo se vinculan con características de los individuos (sexo, edad, origen étnico, nivel formativo, etc.) y con pautas de conducta que pueden ser utilizadas por el empresariado en los procesos de selección. El estatus de los trabajadores en el sistema de reproducción social, tanto en la familia como en la estructura de clases, delimita la posición que ocupan en el mercado de trabajo y define su «nivel de aceptación» de las condiciones laborales, su poder de negociación. La estructura del mercado de trabajo, la posición de los trabajadores y su poder de negociación social cambian a lo largo del tiempo. Son sensibles a las fluctuaciones en el sistema económico que produce cambios en la demanda de empleo de los diferentes segmentos y que modifican las características de los mismos. Esta incidencia de la coyuntura sobre los cambios de estructura se produce de modo más pronunciado en etapas de readaptación y reajuste del mercado de trabajo (Cachón, 1995, 1997). A efectos analíticos, es posible y útil separar las condiciones del mercado de trabajo de las características de los individuos. Sin embargo, estas condiciones tienden a interaccionar entre ellas, lo cual da lugar a diferentes oportunidades de inserción laboral y de integración social, como veremos a continuación. La situación y la dirección de los cambios en el mercado de trabajo de las personas extranjeras se ven afectadas por las modificaciones de ciclo y por los cambios consecuentes, tanto en la demanda como en el poder social de negociación de los trabajadores y en las características de los segmentos del mercado laboral.

En el marco de este debate, en el caso español, el análisis de la cuestión de la calidad del empleo de la población extranjera se ha centrado, al igual que en el plano internacional, en el análisis de cuestiones diversas, como los salarios y las ocupaciones (Martín Artiles et al., 2011; Simón et al., 2008), el sector de actividad (Cachón, 1997) o el régimen de cotización (Carvajal y Pumares, 2004). En este documento y siguiendo la línea abierta por otros estudios (Carrasco, 2008) planteamos la importancia del tipo de contrato como indicador de la calidad del empleo donde se inserta la población extranjera.

Podría argumentarse que la temporalidad tiene significados diferentes en función de, por ejemplo, el sector de actividad o que la creciente flexibilidad ha derivado en una menor estabilidad de los contratos indefinidos y que los temporales pueden encadenarse, lo cual da lugar a relaciones laborales más largas de lo que sugieren los contratos. Martínez (1997) considera la inestabilidad como uno de los rasgos estructurales del mercado de trabajo español. A pesar de ello, el tipo de contrato identifica un estatus reconocido formalmente que podríamos denominar "estabilidad contractual o formal» y que tiene una influencia clara en el trabajador, ya que da lugar a derechos distintos y tiene influencia en la protección del empleo (Laparra, 2006). Asimismo, 
implica diferencias en las condiciones en las que se plantea la relación laboral con el empleador, siendo la situación de quienes tienen contratos temporales de mayor vulnerabilidad que la de los indefinidos. Podemos decir, entonces, que tanto por su influencia en la transformación social, como por su incidencia en otras dimensiones de la calidad del empleo, el análisis del tipo de contrato es un elemento esencial. Aún es más, si bien la cuestión de la continuación en el contrato indefinido puede parecer baladí, ya que su rescisión supone mayores costes que los propios de los temporales, en un contexto de conmoción en el mercado de trabajo como el analizado, es necesario estudiar esta cuestión.

\section{Metodología}

Para el análisis, se ha recurrido fundamentalmente a los ficheros de la Encuesta de Población Activa (EPA) del Instituto Nacional de Estadística (INE). Su finalidad es la obtención de datos de la fuerza de trabajo y de sus diversas categorías (ocupados, parados), así como de la población ajena al mercado laboral (inactivos). La muestra inicial es de 65.000 familias por trimestre. En la práctica, ésta queda reducida a 60.000 familias entrevistadas que equivalen a unas 180.000 personas.

Se han utilizado la versión transversal y los ficheros de flujos EPA. Estos últimos ficheros incorporan una variable que permite vincular registros de la muestra común a lo largo de diferentes trimestres. La encuesta tiene 6 turnos de rotación y cada una de ellas se mantiene en la muestra durante 6 trimestres. Es posible, entonces, vincular distintas proporciones dependiendo de la separación entre trimestres.

Los ficheros de flujos ofrecen información más limitada que los de la EPA trimestral. Algunas variables, como la Clasificación nacional de ocupaciones o la Clasificación nacional de actividades económicas, se ofrecen de manera agregada, a 1 dígito. Otras, que se encuentran disponibles en la versión transversal, no aparecen. Para nuestro análisis, resulta especialmente relevante la imposibilidad de acceder al país de origen o de nacionalidad, así como al tiempo de residencia. Sin embargo, muestra una gran potencialidad para el abordaje de la cuestión que nos ocupa. Permite recoger a la totalidad de situaciones que se dan en la relación con el mercado de trabajo, incluida la inactividad o el desempleo no registrado, independientemente de si tienen cabida en bases oficiales con finalidades administrativas, como el de la Seguridad Social.

La Encuesta de Población Activa Flujos es un panel al uso y, a pesar de sus déficits ya señalados, se optó por proceder a su análisis frente a otras alternativas como la Muestra Continua de Vidas Laborales. Esta última ha sido ya utilizada para analizar la movilidad de la población inmigrante en España (Martín Artiles et al., 2011) y ofrece interesantes posibilidades de análisis, entre otras cuestiones por la amplitud de su muestra. Sin embargo, está construida a partir de conceptos administrativos, lo que obliga a realizar un esfuerzo de comprensión y delimitación de las variables de la muestra como mediciones de conceptos de investigación científica. Los individuos, asimismo, desapare- 
cen si dejan de tener una relación administrativa con la Seguridad Social. Esta cuestión dificulta el seguimiento de colectivos como el extranjero.

Con los ficheros de flujos EPA se han realizado diferentes fusiones. En la primera, se seleccionó a los individuos presentes en el primer trimestre de un año y se les siguió hasta el primer trimestre del año siguiente. En el segundo, con el objetivo de maximizar nuestra muestra, se seleccionó a aquellos individuos presentes, por ejemplo, en el primer trimestre de 2006 con los que estaban presentes en el primer trimestre de 2007. Este ejercicio fue realizado también para el segundo, el tercer y el cuarto trimestre del año. A continuación, se fusionaron las bases de un mismo año. Conseguimos, así, maximizar la muestra para la realización de análisis complejos y controlar por el tiempo en el que se produce el cambio o la permanencia, 12 meses.

En el estudio, recurriremos primeramente a un análisis descriptivo, para centrarnos después en el análisis multinomial. Planteamos un análisis de regresiones logísticas binarias para analizar las probabilidades de movilidad desde el contrato indefinido al desempleo o a la temporalidad. Con las regresiones, se modela la probabilidad de un evento ocurriendo como función de ciertas variables. De esta forma, es posible analizar la importancia relativa de cada variable independiente para explicar el comportamiento de la variable Y. Hemos empleado el sexo, la edad, el nivel de estudios, el tiempo de contrato, el sector de actividad y la cualificación del puesto como variables independientes. El control de estos factores nos ofrecerá una visión compleja de las transiciones y un avance en la identificación de las variables explicativas de las mismas.

\section{El punto de partida. Una reflexión sobre las implicaciones de la destrucción de empleo}

La larga fase de crecimiento económico continuado experimentada en España desde mediados de la década de 1990 fue acompañada de un intenso flujo migratorio que ha tenido consecuencias de muy diverso tipo en el conjunto de la sociedad, algunas de ellas especialmente positivas para el interés colectivo. Sin embargo, como señala Izquierdo (2008), el crecimiento de la economía española durante los últimos tres lustros ha levantado un modelo migratorio que tiende a la exclusión, con una alta proporción de población inmigrante vulnerable, contratada temporalmente en tareas poco valoradas. Dicha vulnerabilidad se ha hecho especialmente patente con el cambio de coyuntura.

El análisis del periodo temporal 2006-2010 muestra que la afección resulta clara en el subcolectivo que trabajaba con contratos temporales, que han sido destruidos en mayor medida. La población extranjera empleada bajo esta modalidad contractual se ha reducido de manera drástica. Se constata, así, un importante cambio por el lado de la demanda. El mercado de trabajo ya no pide ser alimentado, como en la etapa anterior, por los puestos más precarizados. Esto queda reflejado en la reducción del número de ocupados con contratos temporales que se observa, según datos de la EPA, a partir del año 2008 en la población extranjera y en la española. El 58,9\% de la población extranjera 
Tabla 1. Asalariados por nacionalidad y tipo de contrato. Número en miles y porcentaje respecto del total. Población de 16 años o más. Periodo de 2006 a 2010

\begin{tabular}{|c|c|c|c|c|c|c|c|c|c|c|}
\hline & \multicolumn{2}{|c|}{2006} & \multicolumn{2}{|c|}{2007} & \multicolumn{2}{|c|}{2008} & \multicolumn{2}{|c|}{2009} & \multicolumn{2}{|c|}{2010} \\
\hline & \multicolumn{2}{|c|}{ Indefinido Temporal } & \multicolumn{2}{|c|}{ Indefinido Temporal } & \multicolumn{2}{|c|}{ Indefinido Temporal } & \multicolumn{2}{|c|}{ Indefinido Temporal } & \multicolumn{2}{|c|}{ Indefinido Tempora } \\
\hline \multirow[t]{2}{*}{ Español } & 9.727 & 4.056 & 10.172 & 3.929 & 10.461 & 3.730 & 10.503 & 2.957 & 10.219 & 2.771 \\
\hline & 70,6 & 29,4 & 72,1 & 27,9 & 73,7 & 26,3 & 78,0 & 22,0 & 78,7 & 21,3 \\
\hline \multirow[t]{2}{*}{ Extranjero } & 866 & 1.240 & 1.066 & 1.348 & 1.286 & 1.341 & 1.314 & 1.069 & 1.314 & 950 \\
\hline & 41,1 & 58,9 & 44,2 & 55,8 & 49,0 & 51,0 & 55,2 & 44,8 & 58,0 & 42,0 \\
\hline
\end{tabular}

Nota: Información estadística disponible en el anexo.

Fuente: Encuesta de Población Activa. Primer trimestre de cada año.

tenía un contrato temporal en 2006. 4 años más tarde el 42\% se encuentra en esta situación. Ha habido, por tanto, una reducción de 16,9 puntos.

En la población española, se constata que, entre 2009 y 2010, hay 186.000 personas menos ocupadas en puestos temporales. Eso supone una pérdida del 6,31\% de dichos contratos. La bajada de indefinidos es del 2,71\% respecto a los registrados en 2009. En la población extranjera, la reducción de empleos estables no es relevante. En cambio, hay 119.000 personas extranjeras menos con un contrato temporal. Se registra una pérdida del $11,11 \%$ de los contratos de este tipo respecto a 2009.

Asimismo, en el primer trimestre de 2009, se registraban en España 4.026.000 empleos temporales y 11.817.000 indefinidos. Estos datos reflejan una creación, en un momento caótico, de 70.000 empleos indefinidos y la pérdida de 1.045.000 temporales respecto al año anterior. Es destacable que, en el primer trimestre de 2010, se registra ya una reducción generalizada del número de personas asalariadas con independencia del tipo de contrato. Sin embargo, la destrucción de empleo en esta fase de la crisis (hasta 2010) se ha concentrado en los puestos más precarios y temporales. Por tanto, el impacto ha sido menor en los trabajos estables.

Por otro lado, los datos remarcan una importante diferencia en la asignación de los contratos en función de la nacionalidad. Podemos señalar, desde esta perspectiva, que la población inmigrante ha sufrido los efectos más duros de la crisis. El colectivo presenta tasas de temporalidad muy superiores a las de la población autóctona. En consecuencia, la fuerte pérdida de empleo temporal de los últimos años ha ejercido un impacto claro en las personas inmigrantes (Pajares, 2009). Ello viene a poner énfasis en una situación de partida ya de desventaja de la población extranjera, que muestra además altas tasas de desempleo. La pérdida de puestos de trabajo está siendo mucho mayor entre extranjeros que entre nacionales (Muñoz, 2011).

Estudios como el de Carrasco y García (2012) señalan que si bien las tasas de temporalidad de la población extranjera descienden a medida que aumentan los años de residencia en España, no se puede hablar de una situación de asimilación, es decir, de equiparación con los español. En el periodo analizado, las diferencias en la proporción de indefinidos y temporales entre ambas 
poblaciones no bajan de los 20 puntos. La población extranjera muestra bajos niveles de contratación indefinida en comparación con la población española. En el año 2010, el 78,7\% de los asalariados españoles tenía un contrato de este tipo frente al $58 \%$ de la población extranjera.

Aún así, los datos evidencian también que existe un colectivo extranjero importante en términos proporcionales y de volumen que ha conseguido escapar a la trampa de la precariedad contractual, es decir, que tiene un contrato estable (en torno a 1.314 .000 personas extranjeras en 2010). Ello puede ser interpretado como un logro en términos de integración laboral.

Los datos plantean la doble cara de la integración ya desde el inicio. Por un lado, existe un menor impacto de la destrucción de empleo sobre la población empleada bajo contratos indefinidos. Ello resulta de interés, porque 1 de cada 2 trabajadores asalariados extranjeros ha conseguido acceder a dicha situación. Por otro lado, es reseñable que una mayor proporción de población extranjera que de nacional se encuentra en posiciones temporales. Existen importantes diferencias en la integración laboral en función de la nacionalidad.

\section{La movilidad desde la situación de contratación indefinida}

A continuación, analizaremos la movilidad de la población extranjera desde la modalidad de contrato indefinido para entender su situación en el mercado de trabajo. Todo ello se realizará teniendo en cuenta la realidad de la población autóctona, de forma que sea posible la comparación.

Los datos reflejan que la población nacional continúa en contratos indefinidos en mayor medida que la población extranjera. Ésta es una tendencia clara a lo largo del periodo de análisis. Sin embargo, los datos nos muestran también un panorama de fuerte resistencia en el empleo estable entre la población extranjera. Así, el 68,8\% de la población extranjera que en 2006 tenía un contrato indefinido continuaba en dicha situación en 2007. Para el periodo 2009-2010, se constata un aumento de 9,5 puntos en la proporción de personas extranjeras que permanecen en esta situación respecto a 2006-2007. Destaca, entonces, una tendencia a la estabilidad de los estables entre la población extranjera. Entre los nacionales, no se produce un aumento en términos tan marcados. Tanto es así, que se reduce la brecha entre ambas poblaciones en la continuación con un contrato indefinido. En el periodo anterior a la crisis, la diferencia era de 20 puntos y en el último año analizado es de 11 .

Es asimismo reseñable que la población extranjera transita al paro y a la temporalidad en mayor medida. Resulta sin duda llamativo el dato referente a las transiciones a la temporalidad de población extranjera en 2006-2007, que, si bien puede estar sobredimensionado, es coherente con una tendencia a la disminución de ese itinerario en paralelo al aumento de la destrucción de empleo. Para entender la mayor movilidad al desempleo y a la temporalidad de la población extranjera, debemos tener en cuenta que una parte significativa de la población inmigrante ha residido en España durante un periodo de tiempo relativamente corto. El tiempo en el que un individuo se mantiene 
en el mercado de trabajo puede redundar en una mejora de sus condiciones laborales. Asimismo, entendemos que puede relacionarse con el alto índice de rotación de la población extranjera. Es decir, con el hecho de que los extranjeros que permanecen empleados en el mismo sector u ocupación, no lo hacen generalmente para la misma empresa o empleador, lo que les impide beneficiarse de los privilegios tradicionalmente vinculados a la seniority o antigüedad en la empresa dentro del mercado laboral español (Cebolla y González, 2008).

Sin embargo, estas dos transiciones, la de la movilidad hacia la temporalidad y la de la movilidad al desempleo, tienen dos diferencias muy marcadas si se comparan con la población nacional. Por un lado, las diferencias entre ambos colectivos aumentan en referencia a la movilidad hacia el paro. Esta transición era ya protagonizada preferentemente por la población extranjera en 2006-2007. En 2009-2010 lo es en mayor medida, si bien el punto de máxima brecha es en el periodo 2008-2009. Por otro lado, constatamos una reducción del diferencial entre extranjeros y nacionales en la movilidad desde el contrato indefinido hacia la temporalidad. La importante bajada en el porcentaje de personas extranjeras que realizan esta transición, que es mayor que el registrado entre la población española, redunda en una reducción de las distancias. En el periodo 2009-2010, la diferencia en el tránsito a la temporalidad es de 3,8 puntos. Las distancias menos destacables entre la población nacional y la extranjera que se mantiene ocupada se encuentran en el paso de los contratos indefinidos al trabajo por cuenta propia.

A partir del análisis anterior, pueden ser destacados dos tipos de itinerarios con permanencia en la ocupación asalariada: el de los continuos estables, que mantienen un contrato indefinido en los dos periodos analizados, y el de los precarizados, que se mantienen empleados pero en una situación de menos estabilidad de la que comenzaron el año. Es decir, transitaron de un contrato indefinido a uno temporal. Un nuevo ejercicio de cuantificación del número de personas que pertenecen a dichos grupos será realizado a continuación.

La tabla 2 contemplaba, como grupo de mayor interés para entender la transición, a las personas que mantenían una relación con el mercado de tra-

Tabla 2. Transiciones desde la modalidad de contrato indefinido hacia otro tipo de contrato o relación con el mercado laboral por nacionalidad. Población de 16 años o más. Periodo de 2006-2007 a 2009-2010

\begin{tabular}{llcccc}
\hline & & Indefinido & Cuenta propia & Temporal & Desempleado \\
\hline $\mathrm{I} / 2006-\mathrm{I} / 2007$ & Española & 88,7 & 2,9 & 6,7 & 1,6 \\
& Extranjera & 68,8 & 4,2 & 24 & 3,2 \\
$\mathrm{I} / 2007-\mathrm{I} / 2008$ & Española & 89,3 & 2,9 & 5,7 & 2 \\
& Extranjera & 76,7 & 4,4 & 11,7 & 7,1 \\
$\mathrm{I} / 2008-\mathrm{I} / 2009$ & Española & 88,8 & 2,2 & 4,3 & 4,7 \\
& Extranjera & 74,8 & $0,7^{\star}$ & 12 & 12,6 \\
$\mathrm{I} / 2009-1 / 2010$ & Española & 89,3 & 3 & 3,3 & 4,4 \\
& Extranjera & 78,3 & 3,2 & 7,1 & 11,3 \\
\hline
\end{tabular}

* Insuficiencia de la muestra (inferior a 5.000). Información estadística disponible en el anexo.

Fuente: Encuesta de Población Activa (flujos). 
Gráfico 1. Tipologías de transiciones en función del tipo de contrato. Población de 16 a 64 años. Unidades en miles. Periodo de 2006-2007 a 2009-2010
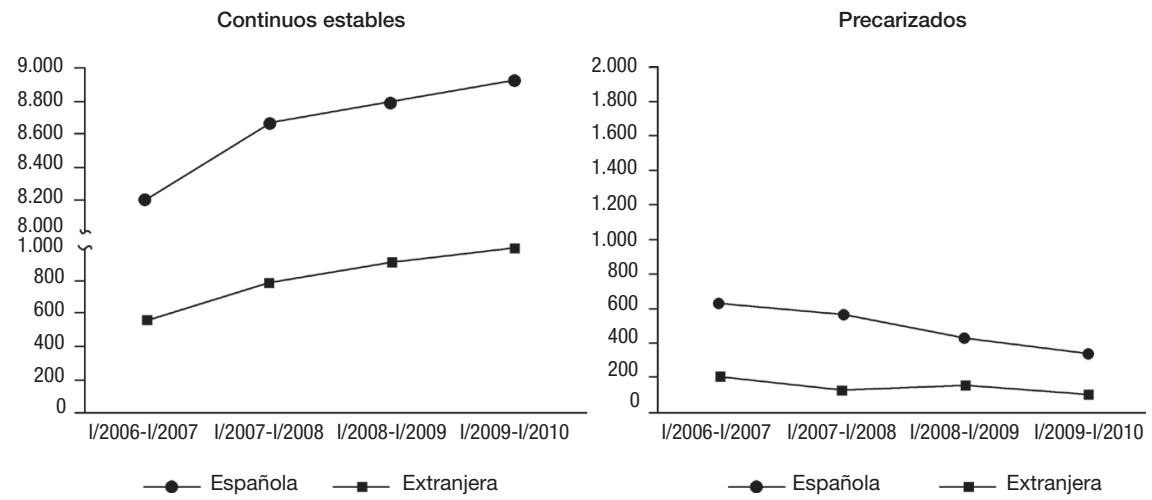

Cálculo realizado mediante la aplicación de los resultados obtenidos en la EPA (flujos) a la EPA transversal. Fuente: Encuesta de Población Activa (flujos) y Encuesta de Población Activa (transversal).

bajo en el punto de destino. Se excluían las transiciones a la inactividad que pueden haber sido realizadas desde la modalidad de indefinido. Este planteamiento, con interés en la EPA flujos, puede sesgar los cálculos sobre la EPA transversal, porque no contempla todas las situaciones de destino posibles. Es por ello que se ha realizado un nuevo análisis sobre la población de 16 a 64 años que, partiendo de un contrato indefinido, transita a cualquier tipo de situación o relación con el mercado de trabajo (contrato indefinido, trabajo por cuenta propia, contrato temporal, desempleo, inactividad). Los datos obtenidos de la EPA flujos han sido aplicados a la EPA transversal para cuantificar el número de personas que pueden ser catalogadas dentro del grupo de los precarizados y de los continuos estables.

Así, se observa un panorama de crecimiento del grupo que denominamos «continuos estables» (mantienen un contrato indefinido en los dos periodos analizados). Es remarcable que el crecimiento del número de estos supervivientes a la crisis, que mantienen una trayectoria positiva, se produce no sólo entre la población nacional, sino también en la población extranjera en un momento crítico en el mercado de trabajo español. Así, en el periodo 2006-2007, se registran 567.712 personas extranjeras que se mantuvieron como indefinidas. En el 2009-2010, el dato asciende a 999.314 personas extranjeras. Por el contrario, se produce una disminución del número de precarizados (transitaron de un contrato indefinido a uno temporal) en ambas poblaciones.

\section{6. ¿Cuál es el perfil de la persona extranjera contratada bajo la modalidad de contrato indefinido?}

En el apartado anterior, avanzamos en la identificación de tipos de transiciones que recorre la población extranjera con incidencia fundamental en su integra- 
ción. Identificamos así la existencia de un colectivo de supervivientes a la crisis que mostraba trayectorias positivas frente a otro protagonista de trayectorias descendentes. Sin embargo, existen importantes diferencias dentro del colectivo extranjero. Como paso previo al análisis de los determinantes de la movilidad, pretendemos establecer el perfil mayoritario del trabajador extranjero estable en España. Analizaremos tanto las características de las personas extranjeras empleadas bajo la modalidad de contrato indefinido como las diferencias en los empleos en los que se insertan.

En el último periodo analizado, encontramos a más mujeres extranjeras que hombres con contrato indefinido. La crisis ha redundado en una inversión de las tornas respecto al sexo. Si bien, hasta el año 2008, eran mayoría los hombres contratados bajo esta modalidad, en 2010, aparece una feminización de la estabilidad del empleo. Esto debe relacionarse con la concentración de la destrucción de puestos de trabajo en sectores donde se insertaban preferentemente los varones extranjeros, como la construcción (Laparra et al., 2012). Es posible, además, que los cambios normativos centrados en la mayor protección de ciertos colectivos como el de las empleadas del hogar, fuertemente feminizado y con importante presencia extranjera, haya tenido una incidencia en este dato. Sin embargo, la muestra con la que trabajamos no nos permite extraer conclusiones a este respecto.

Asimismo, se hace patente que la población extranjera se encuentra todavía hoy caracterizada por una estructura de edad diferente a la nacional. La llegada de población extranjera ha tenido una innegable influencia positiva en el crecimiento de la economía. En informes anteriores a la crisis, como el de Cachón (2004), se constatan tres claves para entenderlo: su estructura de edad que se relaciona con su tasa de actividad superior a la media y, como consecuencia de ella, su elevado nivel de ocupación, que se observa en la tasa de empleo y en el porcentaje de afiliaciones a la Seguridad Social. Sin embargo, dicha población ha sido una de las principales afectadas por el cambio de ciclo y una de las principales víctimas de la destrucción de puestos de trabajo. Es un colectivo joven y esto queda reflejado en el importante peso que el colectivo de 25 a 44 años tiene entre las personas con contrato indefinido. 7 de cada 10 trabajadores indefinidos de nacionalidad extranjera tienen edades comprendidas en dicha franja. Las personas de entre 25 y 34 años son mayoría a lo largo del periodo 2006-2010. Esto difiere de la configuración por edad de la población general (española y extranjera) contratada bajo la modalidad de indefinido, donde destaca el peso de las personas mayores de 44 años. Tanto es así que, en el caso de la población nacional, se hace evidente la precarización que sufren las personas extranjeras menores de 25 años. Son las que muestran mayores dificultades para insertarse en el mercado de trabajo bajo contratos estables. Cabe apuntar, asimismo, que el peso de las personas menores de 25 años es mayor en el colectivo nacional que en el extranjero.

El perfil del trabajador extranjero contratado como indefinido viene determinado por la importancia de las personas con niveles educativos medios y bajos, siendo especialmente relevante la proporción de personas con estudios 
de preprimaria, primaria y ESO. Los trabajadores con niveles educativos altos tienen un menor peso a lo largo del periodo analizado.

Queda claro que las personas extranjeras que se emplean con contratos de tipo estable se insertan preferentemente en puestos cualificados. En el mercado de trabajo español, se registran importantes diferencias en la asignación de las ocupaciones en función de la nacionalidad. Aún así, encontramos a un importante colectivo asentado que ha conseguido acceder a puestos de tipo cualificado y a contratos estables, menos sensibles a los cambios de ciclo como el que se recoge en el periodo analizado. Así, en el año 2010, el 64,6\% de los extranjeros que tenían un contrato indefinido desarrollaban su actividad en ocupaciones de tipo cualificado.

Las condiciones laborales vinculadas al modelo productivo agrícola, caracterizado por una fuerte precarización, ligada a cuestiones como la estacionalidad de las tareas, quedan reflejadas en la baja proporción de personas extranjeras con contratos indefinidos empleadas en el sector de la agricultura. En el año 2010,

Tabla 3. Distribución de las personas extranjeras ocupadas bajo la modalidad de indefinido por sexo, edad, nivel formativo, cualificación del puesto, sector de actividad y tiempo de contrato. Población de 16 años o más. Porcentaje respecto del total. Periodo de 2006 a 2010

\begin{tabular}{|c|c|c|c|c|c|c|}
\hline & & 2006 & 2007 & 2008 & 2009 & 2010 \\
\hline \multirow[t]{2}{*}{ Sexo } & Hombre & 52,6 & 52,1 & 51,2 & 50 & 46,6 \\
\hline & Mujer & 47,4 & 47,9 & 48,8 & 50 & 53,4 \\
\hline \multirow[t]{4}{*}{ Edad } & Menor de 25 años & 9,8 & 10,2 & 9,3 & 8,5 & 7,5 \\
\hline & De 25 a 34 años & 41 & 39,9 & 42,3 & 36,7 & 38,3 \\
\hline & De 35 a 44 años & 30,1 & 31,3 & 30,4 & 35 & 31,6 \\
\hline & Mayor de 44 años & 19,1 & 18,6 & 17,9 & 19,8 & 22,6 \\
\hline \multirow[t]{3}{*}{ Nivel formativo } & Nivel bajo: preprimaria, primaria, ESO & 39,5 & 37,4 & 41,8 & 40,7 & 38,4 \\
\hline & Nivel medio: bachillerato, FP media & 33,3 & 37,5 & 35,2 & 35,8 & 37 \\
\hline & Nivel alto: FP superior, universidad & 27,2 & 25,1 & 23 & 23,5 & 24,6 \\
\hline \multirow{2}{*}{$\begin{array}{l}\text { Cualificación* } \\
\text { del puesto }\end{array}$} & Cualificados & 66,7 & 66,5 & 66,6 & 66,1 & 64,6 \\
\hline & No cualificados & 33,3 & 33,5 & 33,4 & 33,9 & 35,4 \\
\hline \multirow[t]{4}{*}{ Sector de actividad } & Agricultura & 4,7 & 3,5 & 3,4 & 3,7 & 3,7 \\
\hline & Industria & 15,2 & 13,6 & 13,4 & 12,8 & 10,7 \\
\hline & Construcción & 14,7 & 15,5 & 14,3 & 11,8 & 7,1 \\
\hline & Servicios & 65,4 & 67,4 & 68,9 & 71,8 & 78,6 \\
\hline \multirow[t]{4}{*}{ Tiempo de contrato } & Hasta 2 años & 56,9 & 62,4 & 56,1 & 51,1 & 40,8 \\
\hline & De 3 a 4 años & 27,2 & 23,4 & 27,2 & 30,9 & 36,6 \\
\hline & De 5 a 10 años & 11,6 & 10,5 & 13,1 & 13,8 & 18 \\
\hline & Más de 10 años & 4,3 & 3,8 & 3,6 & 4,3 & 4,6 \\
\hline$N$ & & 866.42 & 65.725 & 86.126 & 14.222 & .314 .010 \\
\hline
\end{tabular}

* Siguiendo la Clasificación nacional de ocupaciones-94, englobamos como cualificados a los siguientes grupos: directores de las empresas y de la Administración pública; técnicos y profesionales científicos e intelectuales; técnicos y profesionales de apoyo; empleados de tipo administrativo; trabajadores de servicios de restauración, personales, protección y vendedor de comercio; trabajadores cualificados en agricultura y pesca; artesanos y trabajadores cualificados de industrias manufactureras, construcción y minería; operadores de instalaciones y maquinaria; montadores y trabajadores de fuerzas armadas.

Fuente: Encuesta de Población Activa. Primer trimestre de cada año. 
estas representaban el 3,7\% de los casos. Este dato se encuentra 3,2 puntos por debajo del dato correspondiente al total de población extranjera asalariada empleada en la agricultura (un 6,9\%). En contraposición, destaca la importancia de la población empleada en el sector servicios con contratos estables.

Las personas extranjeras empleadas bajo la modalidad de indefinido con hasta 2 años de tiempo de contrato son mayoría. Es reseñable, además, que, a partir del año 2007, encontramos una tendencia a la baja en la importancia de este colectivo. Si en 2007 representaban el 62,4\% del total de trabajadores no nacionales, en el año 2010, constituyen el 40,8\%. Crece la importancia del resto de subgrupos, más asentados.

El perfil del extranjero contratado bajo la modalidad de indefinido puede establecerse, en el último año analizado, como el de una mujer, de 25 a 34 años, con bajo nivel educativo que se inserta además en puestos con una baja incidencia de la primera fase de la crisis, es decir, en el sector servicios y en ocupaciones cualificadas. Es destacable, además, que, dentro del colectivo de personas con contrato indefinido de nacionalidad extranjera, destacan quienes tienen un contrato de menos de 2 años. Sin embargo, como veremos a continuación, las pautas de movilidad parecen tener sus propios determinantes.

\section{7. ¿Quiénes tienen más probabilidad de perder su contrato indefinido?}

Una vez que hemos repasado la configuración del empleo estable dentro del colectivo extranjero, resulta de interés analizar no sólo cuáles son las características sociodemográficas clave que toman parte en ese colectivo al que hacemos referencia con la etiqueta de población extranjera, sino cómo interactúan entre ellas y con cuestiones vinculadas al puesto de trabajo para explicar las transiciones. A continuación, a través de regresiones logísticas binarias, ponemos en diálogo a las variables utilizadas para el establecimiento del perfil del trabajador extranjero estable. Recurriremos a ellas para el análisis de la movilidad desde el contrato indefinido hasta el desempleo o la temporalidad. El estudio se llevará a cabo para la población extranjera y española.

Existen pautas de movilidad divergentes dentro de los colectivos español y extranjero. En primer lugar, cabe destacar las diferencias por sexo. Hay una desventaja entre las mujeres nacionales y las extranjeras frente a los varones de su misma nacionalidad. Son ellas quienes tienen más probabilidades de perder estos empleos. Las menores diferencias por sexo entre la población no nacional se constatan en el periodo de pleno desarrollo de la crisis del empleo (el periodo 2009-2010). Es reseñable, entonces, que se evidencia una tendencia en esta población a la reducción de la brecha de género. Anteriormente, hemos visto que, en 2010, entre la población extranjera con contrato indefinido son mayoría las mujeres. A esto debemos añadir que, en el último año analizado, prácticamente se puede hablar de una situación de igualdad por sexo en las probabilidades de transición.

En el análisis de la población española y extranjera, los datos confirman hasta 2009-2010 que, a menor edad, más probabilidad de perder el empleo 
indefinido. Esta desventaja se hace patente incluso con el control por el tiempo de contrato. La regresión planteada tiene en cuenta que las personas jóvenes ocupan puestos de trabajo frágiles. No han tenido tiempo de capitalizar su tiempo en el mercado de trabajo en términos de estabilidad mediante un contrato indefinido de mayor duración y con mayores costes de despido. En contraste, no se observa una tendencia clara en relación con el subgrupo de extranjeros con menor probabilidad de pasar al desempleo o a la temporalidad. Los resultados difieren del grupo nacional donde son los mayores de 44 años quienes pierden el empleo indefinido en menor medida. Esto puede ser explicado por el hecho de que sea más probable que pasen a la inactividad y no al desempleo (por ejemplo, con retiros o jubilaciones) (Carbonero et al., 2012).

Esta trayectoria descendente es más probable en los niveles educativos bajos en la población nacional. Las diferencias con los niveles educativos altos, que son quienes tienen menos posibilidades de perder su empleo indefinido a partir del año 2007-2008, experimentan un aumento hasta que, en el periodo 20092010 las distancias se recortan. Esta evolución difiere de la correspondiente a la población extranjera. En este último grupo, los niveles educativos medios y bajos se disputan su preponderancia en la explicación de esta transición. Hasta 2007-2008, en la población extranjera, las personas con niveles medios tienen mayor riesgo de transitar hacia el desempleo o la temporalidad. En los dos años posteriores, en cambio, las posibilidades son mayores si se tiene un nivel educativo bajo. En todo caso, tener un nivel educativo alto supone, en la población extranjera, menos posibilidades de protagonizar esta movilidad. Cabe recordar, además, que las personas con nivel educativo bajo constituyen el colectivo mayoritario dentro de los contratados como indefinidos entre la población extranjera.

El análisis de los grupos nacional y extranjero muestran que las tendencias se asemejan en lo que al sector que implica mayores probabilidades de perder el empleo indefinido se refiere. Éste es la construcción en ambos grupos. Asimismo, queda claro, en el análisis diferencial para la población extranjera y nacional, que tener un trabajo cualificado, a igualdad en otros factores, implica menores posibilidades de perder el empleo indefinido. Por último, es reseñable que son las personas con hasta 2 años de contrato quienes tienen mayor probabilidad de perder su contrato indefinido. Quienes tienen de más de 10 años de contrato corren un riesgo menor.

\section{Conclusiones}

El fenómeno de la temporalidad ha alcanzado un importante desarrollo en el mercado de trabajo español. Hemos asistido a un contexto de creciente fragilidad del empleo, a una expansión de los contratos temporales para todas las categorías laborales. Sin embargo, podemos señalar que el contrato en la modalidad de indefinido supone un estatus reconocido formalmente con implicaciones en el trabajador, entre las que destaca la mayor protección del empleo (Laparra, 2006). Es decir, debe ser vinculada al empleo de calidad. Este artículo tiene como objetivo analizar y comprender las pautas de movilidad de 


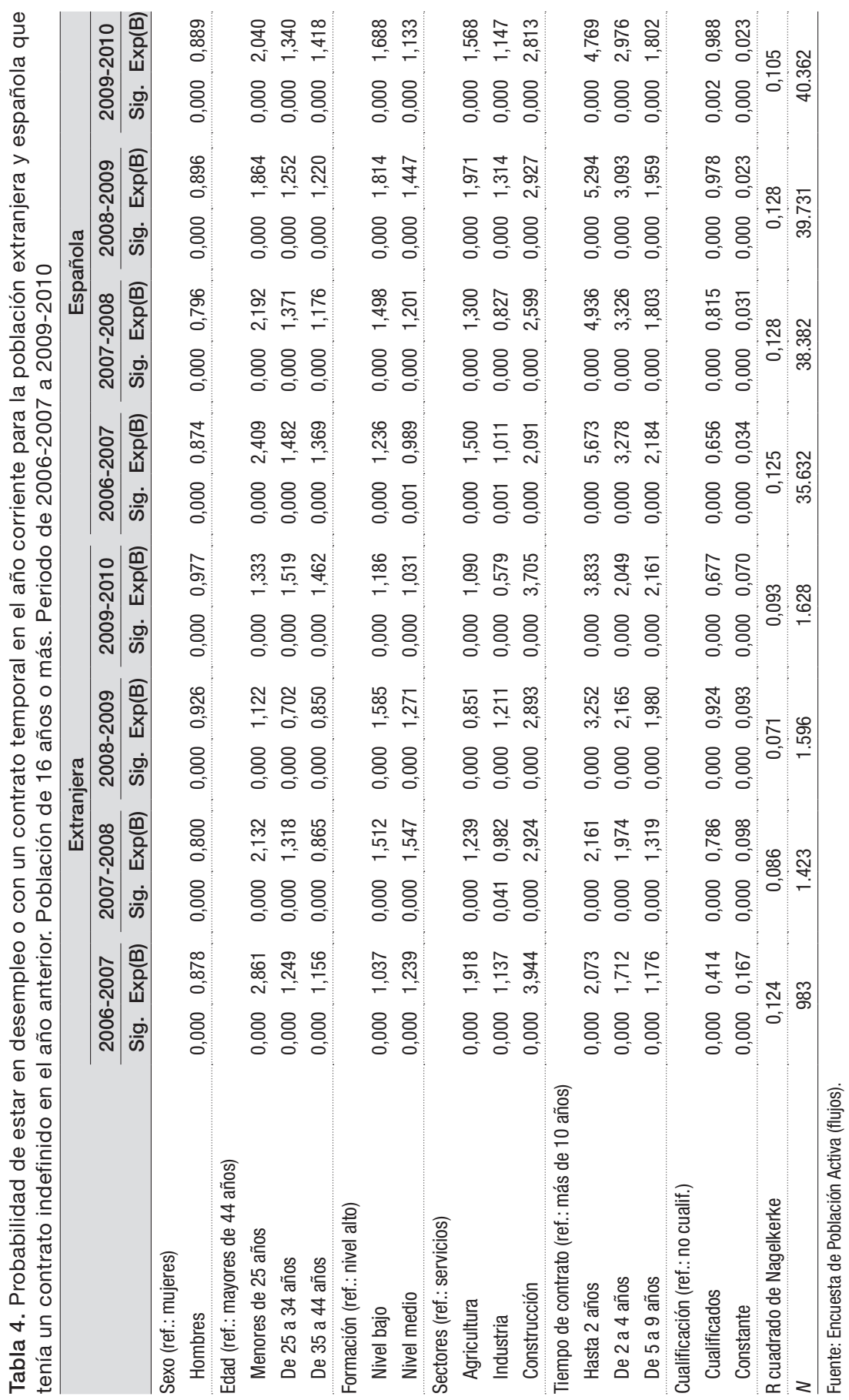


aquellas personas extranjeras que han conseguido acceder a una situación de estabilidad en el empleo, ligada a la contratación indefinida. Hemos recurrido para ello a diferentes herramientas de corte cuantitativo.

El análisis de la distribución de la población ocupada en función del tipo de contrato ha puesto de manifiesto la especial situación de vulnerabilidad de la población extranjera en el contexto de un mercado de trabajo caracterizado por diversos autores como segmentado (Huguet, 1999; Toharia, 1997; Centre d'Estudis Sociològics sobre la Vida Quotidiana i el Treball, 2011). Preferentemente, la población extranjera se ha insertado en puestos temporales, más afectados por la destrucción de empleo. A esto se suman las implicaciones vinculadas al periodo de crisis analizado. Hemos asistido a un cambio por el lado de la demanda de los subsegmentos del mercado de trabajo español, que ya no piden ser alimentados como en la época de bonanza.

Esta situación impacta en las posibilidades de movilidad laboral de las personas extranjeras. Las diferencias en las transiciones desde el contrato indefinido hasta el desempleo entre la población extranjera y la nacional han aumentado drásticamente. Los datos del primer grupo son claramente más negativos. Asimismo, las personas extranjeras transitan a la temporalidad en mayor medida que las personas de nacionalidad española. Esto puede suponer un deterioro en la relación de esta población con la esfera productiva.

Sin embargo, los datos nos muestran también evidencias de la existencia de un subcolectivo extranjero que sobrevive a la crisis. Entre los periodos 20062007 y 2009-2010, se registra un aumento de 9,5 puntos en el porcentaje de personas no nacionales que continúan en la modalidad de contrato indefinido. La proporción de extranjeros que sigue en este tipo de contratos es del 78,3\% en el último año analizado. En la población española, el crecimiento es significativamente más bajo, por lo que el diferencial respecto a los extranjeros disminuye de los 20 puntos en el periodo anterior a la crisis a los 11 del último año analizado. Tanto es así que aumenta el número de personas no nacionales que conserva su contrato indefinido. 1.000 .000 de personas extranjeras se mantienen en dicha situación en el periodo 2009-2010.

Es necesario, entonces, incidir en la importancia de la constatación de dos tendencias: la primera compuesta por aquellos que están cayendo y la segunda por otros que están capeando la crisis. Las personas no nacionales tienen dificultades para acceder al empleo indefinido en el contexto español y, cuando lo consiguen, muestran un mayor riesgo ante la pérdida de dicho empleo. Sin embargo, en un momento en el que el mercado de trabajo se encuentra en estado de conmoción, también se producen resistencias en la integración en el empleo de calidad e incluso aumenta el número de personas extranjeras que consigue mantener su contrato indefinido.

Partiendo de este marco recogido por el análisis de flujo, se ha desarrollado un estudio de los determinantes de la movilidad. Como señalamos en el apartado referente al marco teórico del estudio, existen diferentes oportunidades de empleo y diferentes niveles de aceptación de las condiciones de trabajo entre la población extranjera (Cachón, 1995, 1997). El análisis de regresiones 
nos muestra que no podemos hablar de la integración laboral de la población inmigrante como un proceso único y unidireccional, sino que se trata de un fenómeno complejo en el marco de un modelo migratorio en desarrollo y transformación. Existen diferencias remarcables dentro de ese colectivo al que nos referimos con la etiqueta de "población extranjera». Ser mujer, joven, emplearse en la construcción, tener un trabajo no cualificado y un contrato indefinido de poca duración (hasta 2 años de contrato) aumenta las probabilidades de transitar desde el empleo estable hacia la temporalidad o el desempleo. Tienen, por lo tanto, mayor probabilidad de ser expulsadas de esa posición de relativa ventaja que supone tener un contrato indefinido.

Por otro lado, hemos caracterizado también a ese subcolectivo que permanece asentado en la estabilidad del empleo. El nivel educativo tiene una influencia clara en la posición de la población extranjera en el mercado de trabajo, en este caso, medida a través del contrato indefinido. La inversión en formación se vincula a una integración más estable en el mercado de trabajo. Son las personas extranjeras con un alto nivel formativo, las que tienen menos probabilidades de perder su contrato indefinido. Asimismo, el empleo en un puesto cualificado o la inserción en el mercado de trabajo a través de contratos de muy larga duración (más de 10 años) reducen las probabilidades de pérdida del contrato indefinido.

El plano individual y el estructural se unen, por tanto, en el análisis de la movilidad de la población extranjera. El estudio refleja entonces la complementariedad de los enfoques del capital y de la segmentación a la hora de entender las trayectorias de las personas empleadas bajo la modalidad de contrato indefinido. Tal y como señalan Martín Artiles et al. (2011), ambos enfoques son el anverso y el reverso de una misma moneda.

\section{Referencias bibliográficas}

Althauser, R. P. (1989). «Job histories, career lines and firm internal labor markets». Research on Social Stratification and Mobility, 8, 177-200.

Arango, J. (2003). «La explicación teórica de las migraciones: luces y sombras». Migraciones y Desarrollo, 1, 1-30.

CAchón, L. (1995). «Marco institucional de la discriminación y tipos de inmigrantes en el mercado de trabajo en España». REIS, 65, 105-124. $<$ http://dx.doi.org/10.2307/40183779>

- (1997). «Segregación sectorial de los inmigrantes en el mercado de trabajo en España». Cuadernos de Relaciones Laborales, 10, 49-74.

- (2003). Inmigración y segmentación de los mercados de trabajo en España. Sevilla: CentrA: Fundación Centro de Estudios Andaluces. Serie Sociología.

- (2004). «Integración de los inmigrantes a través del trabajo». Documentación Social, $132,97-120$.

Carbonero, M. A.; Guinea, D. y Zugasti, N. (2012). «Los procesos de exclusión del empleo». En: Laparra, M. y Pérez, B. (coords.). Crisis y fractura social en Europa: Causas y efectos en España. Barcelona: Obra Social "la Caixa". Colección Estudios Sociales, 35. 
Carrasco, C. (2008). "Mercado de trabajo e inmigración». En: Izquierdo, A. (coord.). El modelo de inmigración y los riesgos de exclusión. Madrid: Fundación FOESSA.

Carrasco, M. C. y García, C. (2012). Inmigración y mercado de trabajo. Informe 2011. Madrid: Ministerio de Trabajo e Inmigración. Observatorio Permanente de la Inmigración. Secretaría de Estado de Inmigración y Emigración.

Carvajal, M. I. y Pumares, P. (2004). "Los trabajadores marroquíes en alta laboral en la Seguridad Social». En: Bravo, F.; García, P.; Planet, A. I. y Ramírez, A. (coords.). Atlas de la inmigración marroqui en España. Madrid: Ministerio de Trabajo y Asuntos Sociales.

Cebolla, H. y González, A. (2008). La inmigración en España (2000-2007): De la gestión de flujos a la integración de los inmigrantes. Madrid: Centro de Estudios Políticos y Constitucionales.

Centre d'Estudis Sociològics sobre la Vida Quotidiana i el Treball (2011). Trayectorias laborales de los inmigrantes en España. Barcelona: Obra Social "la Caixa".

Doeringer, P. B. y Piore, M. J. (1971). International Labor Markets and Manpower Analysis. Lexington: D. C. Health and Company.

Fernández, C. y Ortega, C. (2008). "Labor market assimilation of immigrants in Spain: employment at the expense of bad job-matches?». Spanish Economic Review [en línea], 10, 83-107. <http://dx.doi.org/10.1007/s10108-007-9032-4>

Frade, C. y Darmon, I. (2004). Precarious employment in Europe: A comparative study of labour market related risk in flexible economies. Final Report. Barcelona: ICAS Institute.

González, R. y Pérez, B. (2006). "Los efectos de dos décadas de reforma laboral: flexibilidad y precariedad en España». En: LAPARra, M. (coord.). La construcción del empleo precario. Madrid: Fundación FOESSA.

GutiérreZ-Domènech, Maria (2009). «La recesión y el mercado de trabajo». Informe mensual, 326. Barcelona: La Caixa.

Huguet, A. (1999). Segmentación en el mercado de trabajo español. Madrid: Consejo Económico y Social. Colección de Estudios, 76.

Izquierdo, A. (2008). El modelo de inmigración y los riesgos de exclusión. Madrid: Fundación FOESSA. Colección de Estudios, 25.

Kalleberg, A. L. y Sørensen, A. B. (1979). «The sociology of labor markets». Annual Review of Sociology [en línea], 5, 351-379. <http://dx.doi.org/10.1146/annurev.so.05.080179.002031>

Laparra, M. (2006). La construcción del empleo precario: Dimensiones, causas y tendencias de la precariedad laboral. Madrid: Fundación FOESSA.

- (2008). "La dinámica de la integración social de los inmigrantes y su impacto en la sociedad de acogida: La perspectiva desde Navarra». Política y Sociedad, 45 (1), 167-186.

Laparra, M. y PÉrez, B. (2012). Crisis y fractura social en Europa: Causas y efectos en España. Barcelona: Obra Social "la Caixa". Colección de Estudios Sociales, 35.

Laparra, M.; Pérez, B. y Corera, C. (2012). Primer informe sobre desigualdad, pobreza y exclusión social en Navarra: El impacto de la crisis 2007-2011. Estella: Traficantes de Sueños.

Martín Artiles, A.; López-Roldán, P. y Molina, O. (2011). «Movilidad ascendente de la inmigración en España ‘asimilación o segmentación ocupacional?». Papers, $96(4), 1335-1362$. 
Martínez, U. (1997). La integración social de los inmigrantes. Madrid: Trotta.

Massey, D. S.; Arango, J.; Joaquin, H.; Graeme, K.; Kouaouci, A.; Pellegrino, A. y TAYLOR, J. E. (1998). Worlds in Motion: Understanding International Migration at the End of the Millenium. Oxford: Clarendon Press.

Muñoz, J. (2011). «Por qué los trabajadores extranjeros sufren más la pérdida de empleo durante la crisis». Cuadernos de Relaciones Económicas, 225, 147-156.

Pajares, M. (2009). Inmigración y Mercado de Trabajo, Informe 2009. Madrid: Ministerio de Trabajo e Inmigración. Observatorio Permanente de la Inmigración. Secretaría de Estado de Inmigración y Emigración.

Parella, S. (2003). Mujer, inmigrante y trabajadora: La triple discriminación. Rubí: Anthropos.

Pérez Infante, J. I. (2008). «Luces y sombras del mercado de trabajo en España: Problemas, situación y tendencias». Economía Industrial, 367, 35-49.

Piore, M. G. (1983). «El dualismo como respuesta al cambio y a la incertidumbre». En: Toharia, L. (ed.). El mercado de trabajo: Teorias y aplicaciones. Madrid: Alianza Universitaria.

Simón, H.; Sanromá, E. y Ramos, R. (2008). «Labour Segregation and Immigrant and Native-born wage Distributions in Spain: An Analysis using Matched Employer-Employee Data». Spanish Economic Review [en línea], 10 (2), 135-168. <http://dx.doi.org/10.1007/s10108-007-9035-1>

Sørensen, A. B. (1977). "The structure of inequality and the process of attainment». The American Sociological Review [en línea], 42 (6), 965-978. <http://dx.doi.org/10.2307/2094580>

Sørensen, A. B. y Tuma, N. B. (1981). «Labor market structures and job mobility». Research on Social Stratification and Mobility, 1, 67-94.

Toharia, L. (1997). Labour market studies: Spain. Report for the European Commission. Luxemburgo: Office for Official Publications of the European Communities.

Torre, M. (2011). Towards less segregation?: A study of women's occupational mobility in the U. S. labor market. Barcelona: Departamento de Ciencias Políticas y Sociales. Universidad Pompeu Fabra. Tesis doctoral. 


\section{Anexo estadístico}

Tabla 5. Información estadística perteneciente a la tabla 1. Asalariados por nacionalidad y tipo de contrato. Número en miles y porcentaje respecto del total. Población de 16 años o más. Periodo de 2006 a 2010

\begin{tabular}{|c|c|c|c|c|c|c|c|c|c|c|}
\hline & \multicolumn{2}{|c|}{2006} & \multicolumn{2}{|c|}{2007} & \multicolumn{2}{|c|}{2008} & \multicolumn{2}{|c|}{2009} & \multicolumn{2}{|c|}{2010} \\
\hline & Valor & Sig. & Valor & Sig. & Valor & Sig. & Valor & Sig. & Valor & Sig. \\
\hline V de Cramer & 0,212 & 0,000 & 0,212 & 0,000 & 0,196 & 0,000 & 0,188 & 0,000 & 0,171 & 0,000 \\
\hline$N$ válidos & \multicolumn{2}{|c|}{15.888 .950} & \multicolumn{2}{|c|}{16.514 .505} & \multicolumn{2}{|c|}{16.817 .441} & \multicolumn{2}{|c|}{15.843 .068} & \multicolumn{2}{|c|}{15.253 .268} \\
\hline
\end{tabular}

Tabla 6. Información estadística perteneciente a la tabla 2. Transiciones desde la modalidad de contrato indefinido hacia otro tipo de contrato o relación con el mercado laboral por nacionalidad. Población de 16 años o más. Periodo de 2006-2007 a 2009-2010

\begin{tabular}{|c|c|c|c|}
\hline & & Valor & Sig. \\
\hline \multirow[t]{2}{*}{ 2006-2007 } & V de Cramer & 0,158 & 0,000 \\
\hline & $N$ válidos & \multicolumn{2}{|c|}{2.806 .065} \\
\hline \multirow[t]{2}{*}{ 2007-2008 } & V de Cramer & 0,118 & 0,000 \\
\hline & $N$ válidos & \multicolumn{2}{|c|}{ 2.985.357 } \\
\hline \multirow[t]{2}{*}{ 2008-2009 } & V de Cramer & 0,143 & 0,000 \\
\hline & $N$ válidos & \multicolumn{2}{|c|}{3.032 .701} \\
\hline \multirow[t]{2}{*}{ 2009-2010 } & V de Cramer & 0,106 & 0,000 \\
\hline & $N$ válidos & \multicolumn{2}{|c|}{3.090 .377} \\
\hline
\end{tabular}

